\title{
Anaerobic digestion of catering wastes: effect of micronutrients and retention time
}

\author{
M.A. Climenhaga and C.J. Banks
}

School of Civil Engineering and the Environment, University of Southampton, Southampton SO17 1BJ, UK (E-mail: mac2@soton.ac.uk; cjb@soton.ac.uk)

\begin{abstract}
Source-separated foodwastes collected from a campus catering facility were processed in bench-scale single-stage anaerobic digesters. The feedstock contained a varied mix of fruits, vegetables, meats and fried foods. A constant organic loading rate (OLR) was maintained with differing hydraulic retention times (HRT). Regular addition of trace elements or prolonged retention time allowed stable digestion at high total volatile fatty acid (TVFA) levels. Reactors on HRT of 25, 50, and 100 days with no micronutrient supplementation exhibited methanogenic failure after approximately 40, 100 and 90 days respectively, while duplicate reactors with micronutrient supplementation maintained stable digestion. An extended HRT of 180 days has so far allowed continued digestion (for reactors with and without micronutrient supplementation) at levels of ammonia nitrogen exceeding $5.7 \mathrm{gl}^{-1}$ and volatile fatty acid levels exceeding $15 \mathrm{gl}^{-1}$, usually considered inhibitory or toxic.
\end{abstract}

Keywords Anaerobic digestion; catering wastes; micronutrients; foodwastes

\section{Introduction}

Food wastes from domestic kitchens, commercial catering establishments and retail outlets represent a waste stream that holds significant potential as a resource for anaerobic digestion. This study used wastes from an institutional catering facility, containing high quantities not only of readily-degradable starches and sugars, but also more slowly-degradable lipids, as well as proteinaceous materials. The results indicate that a feedstock of pure foodwaste may not contain all nutrients required to meet microbial metabolic requirements.

\section{Methods}

Substrate for the digestion trials was source-separated foodwaste from the main catering facility serving staff and postgraduates at the University of Southampton. Wastes were collected over a period of five days, from all areas of the facility including salad bar (primarily fruit and vegetables), hot food counter (cooked foods including meats and fried foods) and preparation kitchen (peelings, bones and fat trimmings). The wastes were ground in a commercial garbage grinder (S52/010 Waste Disposer, Imperial Machine Company Ltd.) and mixed to form a large composite. The composite was then frozen in $1500 \mathrm{~g}$ portions, stored at $-16^{0} \mathrm{C}$ and thawed as needed for use as substrate. Foodwaste was characterised for total solids (TS), volatile solids (VS) and total Kjeldahl nitrogen (TKN) by standard methods (APHA, 2005). Chemical oxygen demand (COD) was measured following a modification of the standard method (APHA, 2005) using larger quantities of the same reagents to analyse a larger sample.

Eight bench-scale anaerobic bioreactors of 5-litre liquid capacity were operated on a continuous basis for 125 - 200 days (runs of varying length). At the commencement of the trial, sludge from the secondary anaerobic digester of a wastewater treatment plant (Millbrook Sewage Works, Southampton) was collected and sieved through $1 \mathrm{~mm}$ mesh, then used as seed for the reactors. Digestate samples were withdrawn daily and an equivalent quantity of substrate plus distilled water was added to reactors, to maintain a constant volume and fix hydraulic retention time (HRT) in the reactors. A constant organic loading rate (OLR) of $1.45 \mathrm{gVSl}^{-1} \mathrm{~d}^{-1}$ was maintained to all reactors. The reactors were operated on four retention times of 25, 50, 100 and 180 days, with two reactors operating at each retention time. The reactors were run as pairs that were duplicates of each other, except that one reactor from each pair was supplemented with a trace element mixture on a periodic basis ( 1.0 or $1.5 \mathrm{~mL}$ solution weekly, biweekly or monthly depending on HRT) while the other reactor was not. The trace element solution follows the recipe of Gonzalez-Gil et al. (2001). 
Biogas production was measured daily, via water displacement in a volume-calibrated cylindrical gas collector after collection in Tedlar bags. Gas composition samples were withdrawn from the headspace of the reactors once per week, analysed by gas chromatography and compared to a standard mix of $35 \% \mathrm{CO}_{2} / 65 \% \mathrm{CH}_{4}$, on a Varian CP-3800 gas chromatograph with thermal conductivity detector, column temperature $50^{\circ} \mathrm{C}$. Volatile fatty acids were measured weekly using a Shimadzu GC-2010 gas chromatograph, with flame ionisation detector and FFAP capillary column from SGE, model BP-21 $12 \mathrm{~m}$ x $0.32 \mathrm{~mm}$, with helium as carrier gas. Alkalinity measurement and proximate analysis were in accordance with standard methods (APHA, 2005).

\section{Results and Discussion}

The foodwaste contained (mean and standard deviation of five replicates) $\mathrm{TS}=28.1 \pm 0.25 \%$; $\mathrm{VS}=95.5 \pm 0.06 \%$ of TS; TKN=3.77 $\pm 0.24 \%$ of TS; COD $=450 \mathrm{mgg}_{\text {wet weight }}{ }^{-1}$ (single measurement).

Total VFA, methane production, ammonia, alkalinity and Ripley's ratio (Ripley et al., 1986) for each pair of reactors are illustrated in Figures 1-4. The two reactors on a 25-day HRT (Figure 1) show similar VFA and gas production trends at the commencement of the trial, until a steep increase in VFA occurred around Day 40 for the reactor without trace element supplementation. This increase in VFA was associated with a drop in methane production and eventual failure. Up to the time of the failure of the trace element-deprived reactor, the two reactors exhibited very similar behaviour in $\mathrm{pH}$, ammonia, alkalinity and VFA profiles. The supplemented reactor showed stable digestion for three further retention times before also showing a rise in VFA and methane production failure between Days 110-120.

Both reactors in the 50-day HRT pair (Figure 2) had a spike in total VFA production around Day 40-50, but the VFA were consumed over the following weeks. Both reactors exhibited very similar profiles for all parameters measured up to approximately Day 100 (two retention times) at which time the trace element deprived reactor accumulated VFA and ceased to produce methane, while the trace element supplemented reactor maintained stable digestion.

The paired reactors on a 100-day HRT (Figure 3) also show a VFA spike around Day 40-50. The VFA level in the trace element deprived reactor, however, stayed at the higher level, while TVFA in the trace element supplemented reactor was consumed. Toward the end of one retention time (Day 90-100), TVFA in the trace element deprived reactor increased again at the same time as methanogenesis declined.

The paired reactors on a 180-day HRT (Figure 4) were run for over 200 days. Both reactors in the pair have continued to operate through two increases in TVFA. It is interesting to note that the trace element deprived reactor continues to operate in spite of higher VFA concentrations than those at which failure occurred for the reactors on shorter retention times. Reactors on this extended retention time show very high alkalinity (over $18 \mathrm{gl}^{-1}$ ), and are showing stable digestion at a TVFA concentration beyond $15 \mathrm{gl}^{-1}$ and total ammonia nitrogen (TAN) over $5.7 \mathrm{gl}^{-1}$, levels that are often considered to be inhibitory or toxic (Gerardi, 2003, Grady et al., 1999). This trial is continuing, to investigate long-term stability.

The increases in VFA around Day 40 and Day 100 are observed for all reactors. This cannot be attributed to external or equipment factors because the runs were commenced on different dates months apart, and therefore Day 40 for the 25-day HRT reactors falls on a different calendar date than Day 40 for the 50- and 100-day HRT reactors or the 180-day HRT reactors. Additionally, the phenomenon was observed on three previous digestion trials with HRT of 25 and 50 days (data not shown). Therefore, it is a phenomenon attributable to factors internal to the system. As the reactors are on the same OLR, at Days 40 and 100 all will have received the same cumulative VS, 


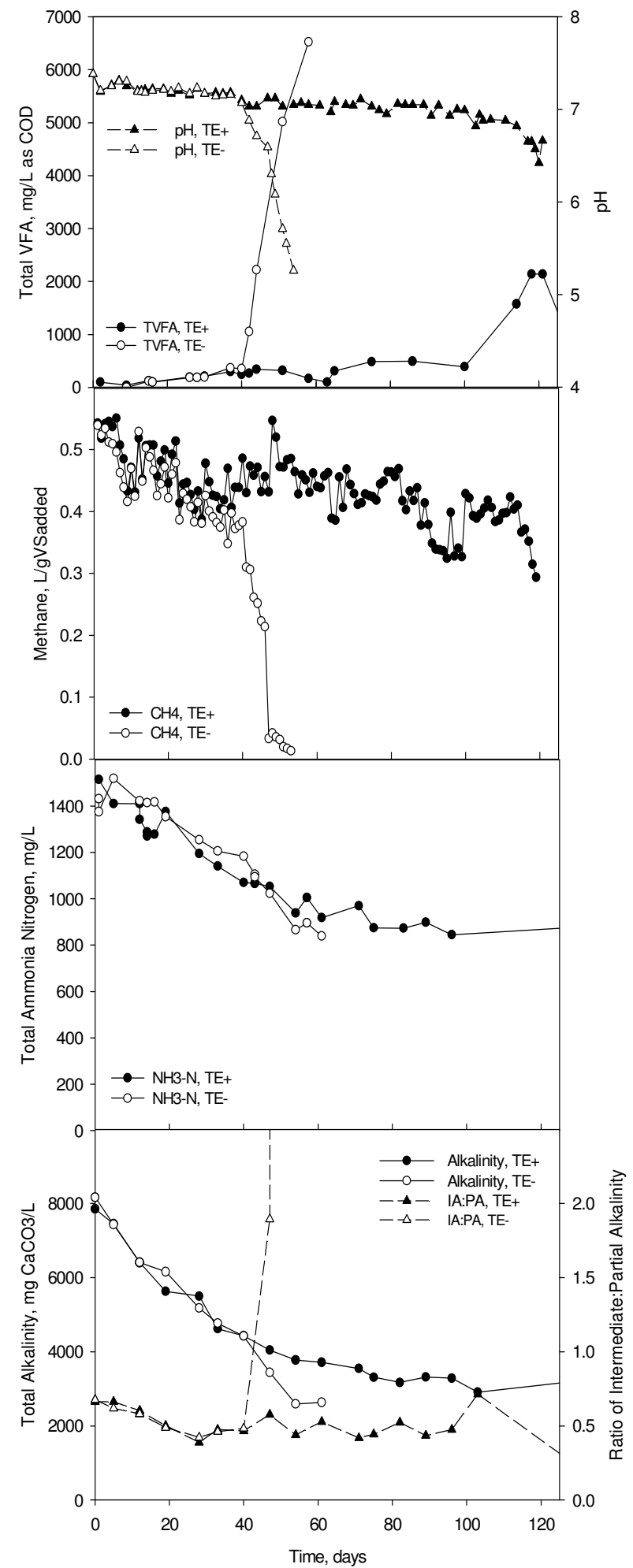

Figure 1 TVFA and $\mathrm{pH}, \mathrm{CH}_{4}$ production, TAN, total alkalinity and alkalinity ratios for reactors with (TE+) or without (TE-) trace element supplementation, $\mathrm{HRT}=25$ days.

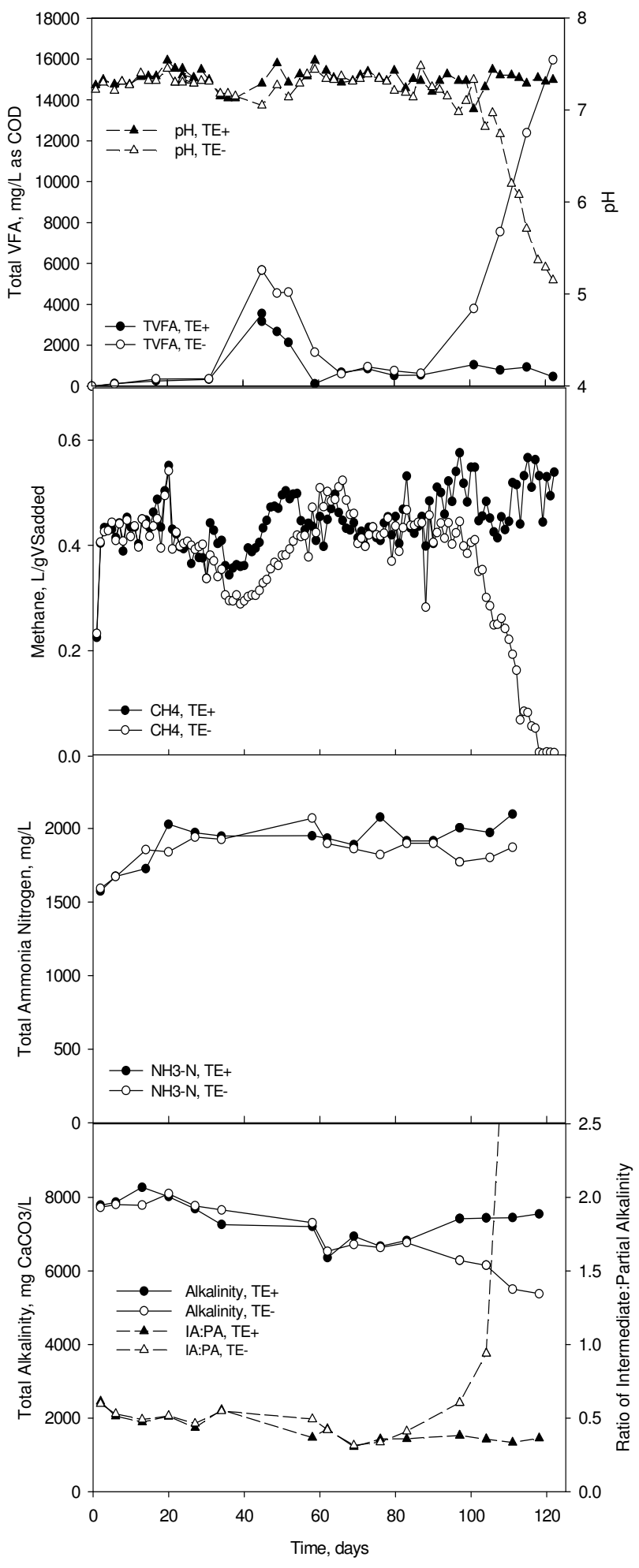

Figure 2 TVFA and $\mathrm{pH}, \mathrm{CH}_{4}$ production, TAN, total alkalinity and alkalinity ratios for reactors with (TE+) or without (TE-) trace element supplementation, $\mathrm{HRT}=50$ days.

although the rate of washout and therefore accumulation of breakdown products such as ammonia differs for the different retention times. 


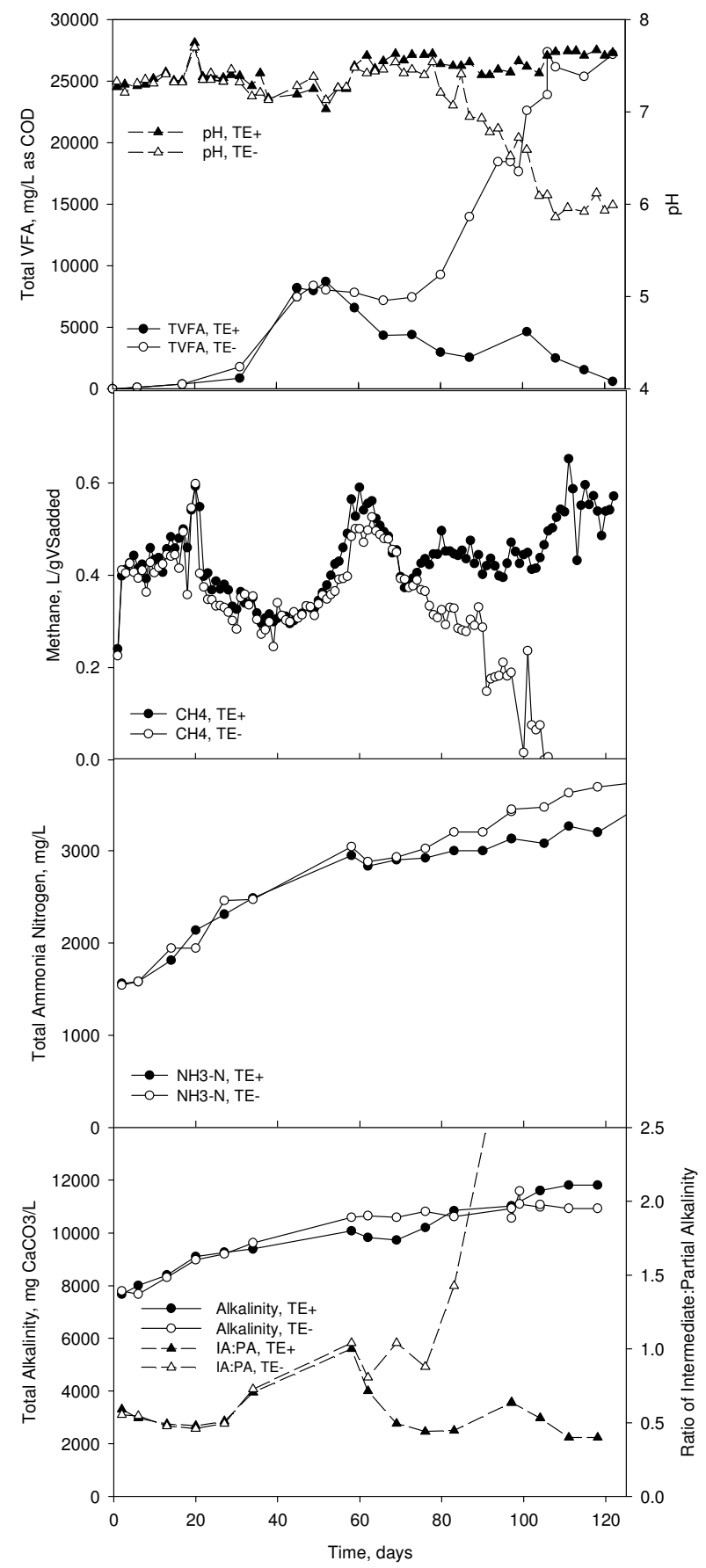

Figure 3 TVFA and $\mathrm{pH}, \mathrm{CH}_{4}$ production, TAN, total alkalinity and alkalinity ratios for reactors with (TE+) or without (TE-) trace element supplementation, $\mathrm{HRT}=100$ days.

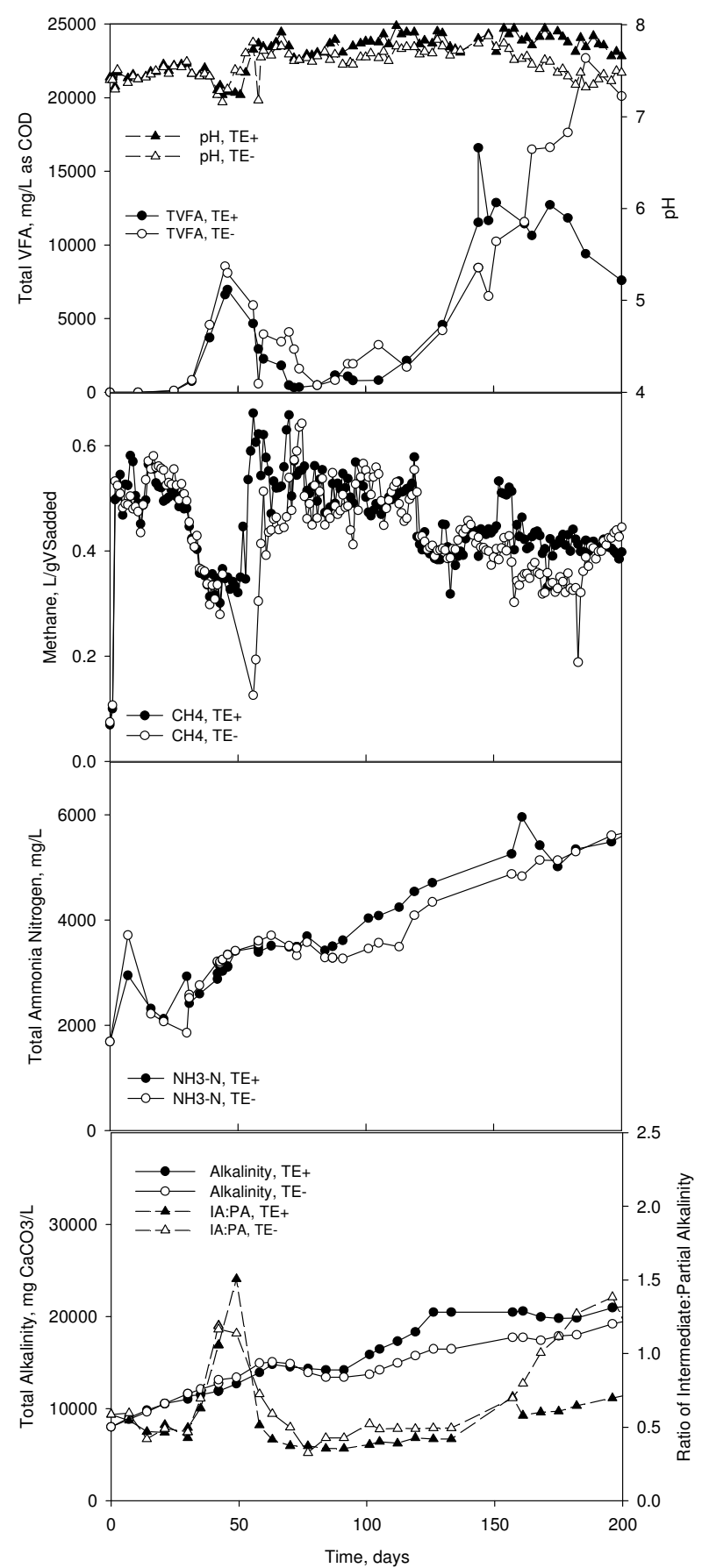

Figure 4 TVFA and $\mathrm{pH}, \mathrm{CH}_{4}$ production, TAN, total alkalinity and alkalinity ratios for reactors with (TE+) or without (TE-) trace element supplementation, $\mathrm{HRT}=180$ days.

Role of Trace Elements: A number of investigators have pointed to the importance of certain trace elements such as iron, cobalt, and nickel for maintaining a stable anaerobic digestion process (Florencio et al., 1994, Zandvoort et al., 2002), but these studies have generally been focused on a simple and defined feedstock such as methanol. The addition of trace elements, nutrients or alkalinity is commonly practiced in many laboratory anaerobic digestion studies on wastewaters that are known to be deficient in certain nutrients (Takashima and Speece, 1990), or in batch studies, but in many cases no addition of other compounds is practiced, especially on studies with continuous feeding of diverse mixed feedstocks (Carucci et al., 2005, Fernandez et al., 2005, 
Salminen and Rintala, 2002). Also, micronutrient supplementation in commercial operations is not commonly practised as the minimisation of costs is favoured. The feedstock studied in this investigation is a complex waste containing a wide range of foods, which might be expected to contain sufficient amounts of all trace elements required for cell metabolism. It is possible, however, that either the feedstock lacks all of the trace elements specifically required for methanogenic metabolism, or that the elements are present but not bioavailable. Heavy metals are known to be precipitated by sulphides (Gerardi, 2003); long-chain fatty acids (LCFA) can also bind with minerals such as calcium (Pereira et al., 2001). Therefore although the substrate contains a broad range of nutrients and may contain all of the trace elements required, these may become nonbioavailable during the anaerobic digestion process.

A second possibility for the role of micronutrients is in support of biomass resistance to inhibition or toxicity by LCFA or other inhibitory substances in the medium. Other investigators have found an effect of micronutrient supplementation under unfavourable conditions; for example a mix of iron, nickel and cobalt was shown to play a role in supporting maintenance of granular sludge in UASB reactors treating food processing wastewater (Oleskiewicz, 1989) and in other work the presence of substoichiometric amounts of ferric hydroxide reduced the sensitivity of acetoclastic methanogenesis to inhibition by fatty acids in anaerobic sediments incubated with vegetable oil ( $\mathrm{Li}$ et al., 2005). Whether this is due to precipitation of LCFA by metals, thereby lessening their inhibitory effect, by direct support to microorganisms or by some other mechanism, is unknown and is being investigated further.

Role of Ammonia / Ammonium: The 100-day HRT reactors sustained total ammonia nitrogen (TAN) levels beyond $3 \mathrm{gl}^{-1}$, while in the 180-day HRT reactors TAN currently exceeds $5.7 \mathrm{gl}^{-1}$ at a $\mathrm{pH}$ above 7.5. Free ammonia, as calculated following Kaparaju and Rintala (2005), therefore exceeds $1000 \mathrm{mgl}^{-1}$, beyond the threshold of $300 \mathrm{mgl}^{-1}$ observed to have a severe inhibitory effect (Angelidaki and Ahring, 1993). In this investigation, TAN appears to be more beneficial than detrimental, as it provides buffering capacity in the long HRT reactors, as opposed to the 25-day HRT reactors in which TAN is washed out and declines through the trial. Bhattacharya and Parkin (1989) reported digestion in chemostat cultures fed on acetate with addition of up to $5 \mathrm{gl}^{-1} \mathrm{TAN}$, but failure at $6 \mathrm{gl}^{-1}$ TAN. This was observed with cultures on a 40-day retention time, while at lower retention times failure was observed at lower TAN concentrations; in this study, extending retention time to 180 days has allowed stable digestion at over $5.7 \mathrm{gl}^{-1}$ TAN. In thermophilic digestion of cattle manure, $4 \mathrm{gl}^{-1}$ TAN was found to be inhibitory, but acclimation over 6 months of operation allowed digestion at up to $6 \mathrm{gl}^{-1}$ TAN, with reduced activity and a VFA concentration of $3 \mathrm{gl}^{-1}$ as acetate (Angelidaki and Ahring, 1993); in this study, TVFA has exceeded $15 \mathrm{gl}^{-1}$ as COD in a reactor that continues to operate. This also agrees with previous findings of pilot-scale $(1500 \mathrm{~L})$ single-stage digestion of nitrogen-rich foodwastes, in which system alkalinity was high and stable digestion at VFA levels up to $25 \mathrm{gl}^{-1}$ was maintained (Stringfellow et al., 2003)(Stringfellow et al., in press).

\section{Conclusions}

This work has shown the importance of micronutrients in digestion of a mixed foodwaste feedstock. Reactors supplemented with trace elements showed stable digestion, while non-supplemented reactors showed methanogenic failure. Also, the extended retention time trial has shown an interesting result in the maintenance of stable digestion in spite of elevated concentrations of VFA (over $15 \mathrm{gl}^{-1}$ ) and total ammonia nitrogen (over $5.7 \mathrm{gl}^{-1}$ ). In addition to the role of micronutrients, system resilience at extended retention times is currently being investigated further. Current and future work will focus on the mechanisms by which trace elements act to support stable digestion, and determination of the relative importance of the retention of soluble compounds versus the retention of biomass. 


\section{Acknowledgements}

This work was supported by funding from the UK Engineering and Physical Sciences Research Council (EPSRC) Sustainable Urban Environment (SUE) Programme grant GR/S79626/01, and the UK Overseas Research Students Awards Scheme (ORSAS). The invaluable assistance of Denise Cysneiros, Mark Walker, Sonia Heaven and Yue Zhang are gratefully acknowledged.

\section{References}

Angelidaki, I. and Ahring, B. K. (1993). Thermophilic anaerobic digestion of livestock waste: the effect of ammonia. Environmental Biotechnology, 38(4), 560-564.

Standard Methods for the Examination of Water and Wastewater (2005). 21st edn, APHA, (American Public Health Association), American Wastewater Association and Water Environment Federation, Washington, D.C.

Bhattacharya, S. K. and Parkin, G. F. (1989). The Effect of Ammonia on Methane Fermentation Processes. Journal of the Water Pollution Control Federation, 61(1), 55-59.

Carucci, G., Carrasco, F., Trifoni, K., Majone, M. and Beccari, M. (2005). Anaerobic digestion of food industry wastes: Effect of codigestion on methane yield. J. Environ. Eng.-ASCE, 131(7), 10371045.

Fernandez, A., Sanchez, A. and Font, X. (2005). Anaerobic co-digestion of a simulated organic fraction of municipal solid wastes and fats of animal and vegetable origin. Biochemical Engineering Journal, 26(1), 22-28.

Florencio, L., Field, J. A. and Lettinga, G. (1994). Importance of Cobalt for Individual Trophic Groups in an Anaerobic Methanol-Degrading Consortium. Applied and Environmental Microbiology, 60(1), 227-234.

Gerardi, M. H. (2003). The Microbiology of Anaerobic Digesters, John Wiley \& Sons, Inc., Hoboken, New Jersey.

Gonzalez-Gil, G., Seghezzo, L., Lettinga, G. and Kleerebezem, R. (2001). Kinetics and MassTransfer Phenomena in Anaerobic Granular Sludge. Biotechnol. Bioeng., 73(2), 125-134.

Grady, C. P. L., Daigger, G. T. and Lim, H. C. (1999). Biological Wastewater Treatment, Marcel Dekker, New York.

Kaparaju, P. and Rintala, J. (2005). Anaerobic co-digestion of potato tuber and its industrial byproducts with pig manure. Resources, Conservation and Recycling, 43, 175-188.

Li, Z. K., Wrenn, B. A. and Venosa, A. D. (2005). Effect of iron on the sensitivity of hydrogen, acetate, and butyrate metabolism to inhibition by long-chain fatty acids in vegetable-oil-enriched freshwater sediments. Water Research, 39(13), 3109-3119.

Oleskiewicz, J. A. (1989). Granulation in anaerobic sludge bed reactors treating food industry wastes. Biol. Wastes, 27, 217-235.

Pereira, M. A., Mota, M. and Alves, M. M. (2001). Degradation of oleic acid in anaerobic filters: the effect of inoculum acclimatization and biomass recirculation. Water Environment Research, 73, 612-621.

Ripley, L. E., Boyle, W. C. and Converse, J. C. (1986). Improved alkalimetric monitoring for anaerobic digestion of high-strength wastes. Journal WPCF, 58(5), 406-411.

Salminen, E. A. and Rintala, J. A. (2002). Semi-continuous anaerobic digestion of solid poultry slaughterhouse waste: effect of hydraulic retention time and loading. Water Research, 36(13), 31753182.

Stringfellow, A., Banks, C. J., Pritchard, L. J. and Chesshire, M. J. (2003). Survival of Salmonella in the anaerobic digestion of kitchen waste. Proceedings of the ninth international waste management and landfill symposium, S Margherita di Pula (Cagliari), Sardinia, Italy. Takashima, M. and Speece, R. E. (1990). Mineral Requirements for Methane Fermentation. Critical Reviews in Environmental Control, 19(5), 465-479.

Zandvoort, M. H., Geerts, R., Lettinga, G. and Lens, P. N. L. (2002). Effect of long-term cobalt deprivation on methanol degradation in a methanogenic granular sludge bioreactor. Biotechnology Progress, 18(6), 1233-1239. 